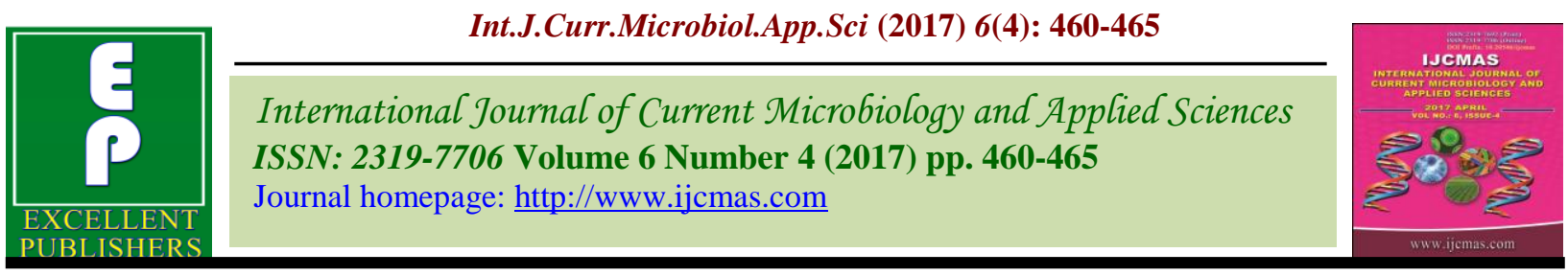

Original Research Article

https://doi.org/10.20546/ijcmas.2017.604.053

\title{
Screening of Carbapenem Resistant Enterobacteriaceae among Nosocomial Isolates: A Study from South India
}

\author{
T.V. Parimala* \\ Department of Microbiology, Shridevi Institute of Medical Sciences and Research Hospital, \\ NH4, Sira road, Tumkuru, Karnataka, India \\ *Corresponding author
}

\section{A B S T R A C T}

\section{Keywords}

Antibiotic resistance, Carbapenem,

Enterobacteriaceae, Carbapenem resistant Enterobacteriaceae, Nosocomial isolates.

Article Info

Accepted:

02 March 2017

Available Online:

10 April 2017
Enterobacteriaceae are inhabitants of the intestinal flora. Emergence of Carbapenem resistant Enterobacteriaceae (CRE) is a serious threat to public health. They rapidly acquire and transfer drug resistance genes through plasmids and transposons and spread to community via faeco-oral route. Hence the present study was done to know the magnitude of Carbapenem resistant Enterobacteriaceae in our hospital. This study was conducted in the department of Microbiology, SIMS and RH, Tumkuru during a period from May 2016 to October 2016. A total of 106 Enterobacteriaceae isolates were obtained by various clinical samples and from different department of the hospital. These isolates were screened for carbapenem resistance by disk diffusion method according to CLSI guidelines. Prevalence of CRE in our hospital is 44. 33\%. Meropenem resistance (31. $13 \%$ ) was higher than Imipenem resistance among CRE isolates. In this study, CRE is more common in male $(50.90 \%)$ patients. Majority of the sample is urine $(55.26 \%)$ and Maximum number of cases was from (51.61\%) OBG department. Most common species isolated was (63. 04\%) Escherichia coli. Carbapenem resistant Enterobacteriaceae are more prevalent in our hospital. Hence use of carbapenem should be restricted to treat severe infections.

\section{Introduction}

Enterobacteriaceae is a family of Gram negative bacilli which can cause a wide range of community acquired and nosocomial infections including infections of the urinary and gastrointestinal tracts, pneumonia, peritonitis, meningitis, sepsis and medical device associated infections (Surinder Kumar, 2012; Wang et al., 2015; Harbaik et al., 2014). These organisms easily acquire and transfer drug resistance genes through plasmids and transposons (Okoche et al., 2015). Carbapenem are beta lactam antibiotics and are the last resort for the treatment of severe infections caused by multidrug resistant Gram negative bacilli (Gupta et al., 2006; Roy et al., 2011). Reports indicate that carbapenemases producing Enterobacteriaceae (CRE) isolates seem to be increasing in number in the last few years (Nagaraj et al., 2012).

Resistance to carbapenem is mostly due to the production of carbapenemases, which are beta lactamase enzymes with a capacity to hydrolyze not only the carbapenem but also all other beta lactam agents (Mate et al., 
2014). It can also be due to poor binding to carbapenem to pencillin binding protein present in the bacteria, the over expression of multidrug efflux pumps by the bacterial cell membrane (Nair et al., 2013). Infections caused by CRE have limited treatment options and have been associated with high mortality rates ranged from $22 \%-72 \%$ (Wang et al., 2015 and Weinstien et al., 2011). Appropriate detection of $\mathrm{CRE}$ is vital for patient care in order to institute correct therapeutic options (Datta et al., 2012). Hence present study was done to determine the magnitude of carbapenem resistant Enterobacteriaceae in our hospital.

\section{Materials and Methods}

This prospective study was carried out in the clinical bacteriology, in the Department of Microbiology at Shridevi Institute of Medical sciences and Research Hospital, Tumkuru during a period from May 2016 to October 2016. A total of 106 clinical isolates belong to Family Enterobacteriaceae from various clinical samples were included. The distribution of the sources of the isolates were pus, urine, blood, stool, sputum and ET secretions from different wards of hospital, outpatient departments and ICUs.

All the isolates were identified as per the standard microbiological procedure and stocked in $0.2 \%$ semisolid agar until analyzed. These isolates were screened for their ability to produce carbapenamases on Muller Hinton Agar plates by Kirby - Baurer disk diffusion method as per CLSI guidelines using Imipenem (10ug) and Meropenem (10ug) disk. Both antibiotic discs were procured from Hi-media, Mumbai.

The diameter of the zones of inhibition of growth was recorded and sensitive, intermediate and resistant based on CLSI guidelines. Organisms with intermediate levels of resistance to the antibiotics were included in the resistant organisms for final analysis. Data were analysed with Microsoft excel.

\section{Results and Discussion}

A total of 106 Gram negative bacilli belong to Enterobacteriaceae family, were isolated during the study period. Among 106 clinical isolates, $47 \mathrm{CRE}$ isolates showed resistance to carbapenem by the disk diffusion method. Out of 47 (44. 33\%) CRE isolates, 33 (31. 13\%) were resistant to Meropenem and 14 (13. $20 \%$ ) were resistance to Imipenem (Table 1). CRE isolates more commonly seen in 28 (50. 90\%) male patients than 19 (37. 25\%) female patients (Table 2). CRE organisms most frequently isolated on microbiological cultures of $21(55.26 \%)$ urine samples followed by 18 (54. 54\%) pus samples (Fig 1), majority of the CRE isolates were from 16 (51. 61) OBG department next was 14 (46. 66\%) medicine department. (Fig 2) The different strains of CRE isolates were Escherichia coli, Klebsiella spp, Enterobacter spp, Citrobacter spp, Proteus spp, Providencia spp and Shigella. Maximum number of CRE isolates in this study was 21 (55. 26\%) Escherichia coli (Table 3).

Microbial drug resistance is a growing problem of global magnitude (Praveen et al., 2010. Emergence and dissemination of Carbapenem resistance among Enterobacteriaceae poses a considerable threat to public health and thought to be due to high antibiotic use and misuse without proper diagnosis of infection or self medication by patients (Okoche et al., 2015; Birgy, 2012). Additionally, other factors may include co selection by other antibiotics (e. g. cephalosporins), spread of those bacteria in community, uncontrolled spread in the hospital environment, owing to undiagnosed carriers and other, still unknown, factors 
(Harbaik et al., 2014). The detection of Carbapenems producers in clinical infections is based first on susceptibility testing results obtained by disk diffusion / by automated systems (Nordaman et al., 2011). In the present study 33 (31. 13\%) were more resistant to Meropenem than to14 (13. 20\%) Imipenem. Similarly, study conducted by Temple et al., Meropenem resistance (57\%) was higher than Imipenem resistance (49. $5 \%$ ). This may be due to increased used of meropenem in this area (Temple et al., 2015).

Table.1 Resistance to carbapenem among Gram negative bacilli belong to Family

\section{Enterobacteriaceae}

\begin{tabular}{|l|l|l|l|l|l|l|}
\hline Antibiotic & $\begin{array}{l}\text { Carbapenem } \\
\text { resistant }\end{array}$ & percentage & $\begin{array}{l}\text { Carbapenem } \\
\text { sensitive }\end{array}$ & percentage & Total & percentage \\
\hline Imipenem & 14 & 13.20 & 92 & 86.79 & 106 & 100 \\
\hline Meropenem & 33 & 31.13 & 73 & 68.86 & 106 & 100 \\
\hline Total & 47 & 44.33 & 59 & 55.66 & 106 & 100 \\
\hline
\end{tabular}

Table.2 Gender wise distribution of CRE isolates

\begin{tabular}{|l|l|l|l|l|l|l|}
\hline Gender & $\begin{array}{l}\text { Carbapenem } \\
\text { resistant }\end{array}$ & percentage & $\begin{array}{l}\text { Carbapenem } \\
\text { sensitive }\end{array}$ & percentage & Total & percentage \\
\hline Male & 28 & 50.90 & 27 & 49.09 & 55 & 51.88 \\
\hline Female & 19 & 37.25 & 32 & 62.74 & 51 & 48.11 \\
\hline Total & 47 & 44.33 & 59 & 55.66 & 106 & 100 \\
\hline
\end{tabular}

Table.3 Distribution of species of CRE isolates

\begin{tabular}{|c|c|c|c|c|c|c|}
\hline Species & $\begin{array}{l}\text { Carbapenem } \\
\text { resistant }\end{array}$ & percentage & $\begin{array}{l}\text { Carbapenem } \\
\text { sensitive }\end{array}$ & percentage & Total & percentage \\
\hline $\begin{array}{l}\text { Escherichia } \\
\text { coli }\end{array}$ & 29 & 63.04 & 17 & 36.95 & 46 & 43.39 \\
\hline $\begin{array}{l}\text { Klebsiella } \\
\text { spp }\end{array}$ & 12 & 42.85 & 16 & 57.14 & 28 & 26.41 \\
\hline $\begin{array}{l}\text { Enterobacter } \\
\text { spp }\end{array}$ & 03 & 27.27 & 08 & 72.72 & 11 & 10.37 \\
\hline $\begin{array}{l}\text { Citrobacter } \\
\text { spp }\end{array}$ & 01 & 10.00 & 09 & 90.00 & 10 & 9.4 \\
\hline Proteus spp & 01 & 16.66 & 05 & 83.33 & 06 & 05.66 \\
\hline $\begin{array}{l}\text { Providencia } \\
\text { spp }\end{array}$ & 01 & 25.00 & 03 & 75.00 & 04 & 03.77 \\
\hline Shigella spp & 00 & 00 & 01 & 100 & 01 & 00.94 \\
\hline Total & 47 & 44.33 & 59 & 55.66 & 106 & 100 \\
\hline
\end{tabular}


Fig.1 Sample distribution of CRE isolates

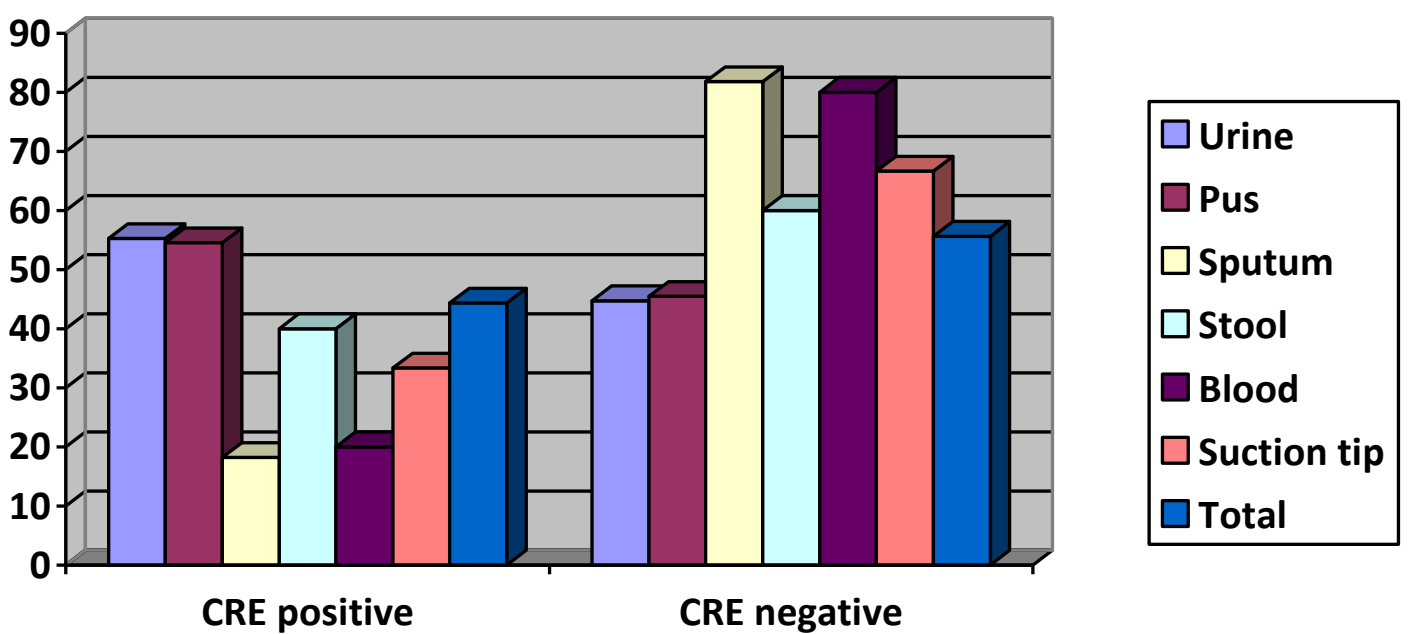

Fig.2 Distribution of CRE isolates in different department of hospital

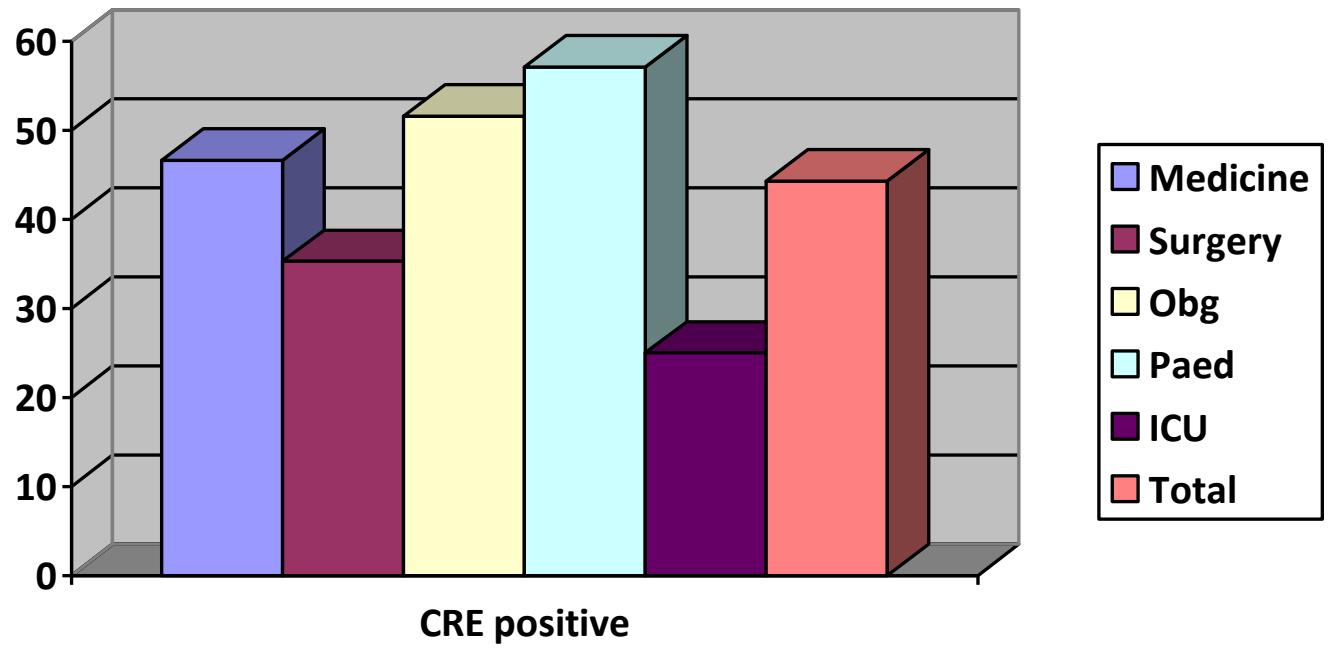

Maximum number of CRE isolates was from $21(55.26 \%)$ urine sample followed by pus sample (55.54\%), sputum (18.18\%) and others observed in our study. Nagaraj et al., (2012) observed that carbapenem-resistent organism were isolated mainly from urine samples up to $42 \%(\mathrm{n}=21)$, followed by wound discharge $18 \%$ and respiratory secretions $16 \%$. The reason for this could be urinary tract infection (UTI), being the most common hospital acquired infection which accounts for almost $40 \%$ of all nosocomial infections (Mate et al., 2014).
The most common species among CRE isolates was E. coli $(63.04 \%)$ in the present study. Wattal et al., (2010) reported high prevalence of resistance to carbapenems ranging from 13 to $51 \%$ in E. coli and Klebsiella spp. The actual prevalence of carbapenemase producers is still unknown because many countries that are likely to be their main reservoirs have not established any search protocol for their detection (Nordaman et al., 2011). In the present study, prevalence of CRE is $44.33 \%$ which is higher compared to other studies. Gupta et al., (2006) also 
reported high prevalence of resistance varying from 17 to $22 \%$ to various carbapenems among Enterobacteriaceae strain. Datta et al., (2012) found that the prevalence of CRE in their Institute is 7. 87. In the present study of prevalence of CRE is $44.33 \%$ which is higher compared to other studies (Mate et al., 2014 and Datta et al., 2012).

Center for disease control has laid down guidelines for prevention of CRE infection (CDC guidelines, 2014). It includes 8 core measures which have been included in the prevention strategies and include laboratory detection; rigorous implementation and monitoring of infection control measure such as hand hygiene, contact precautions, isolation, setting up of ICC, proper medical waste disposal, and maintenance of environmental hygiene; education of training of health care personnel on proper use and rationale of infection control measures along with appreciation/reward of units/staff for best infection control; Minimal use of invasive devices; antibiotic stewardship; effective and rational dosing of antibiotics integrating pharmacokinetic and pharmacodynamic profiles of the antibiotic with the microbiological data (minimal inhibitory concentration); screening (rectal and perirectal) sampling of epidemiology linked contact of colonised or infected patients, active surveillance; and coordinated effects among health care faculties and encouragement of antimicrobial research and development.

In conclusion the present study indicates the prevalence of Carbapenem resistant Enterobacteriaceae was high (44. 33\%) compared to other studies. Hence, strict implementation of infection control of policies is required to control the spread of CRE infection and Carbapenem should be used as reserve drug in the treatment of severe gram negative infections. Also, it is advisable to use those agents such as colistin, tigecyline, fosfomycin as salvage therapy in treatment of MDR and PanDR resistance organism.

\section{References}

Birgy, A., Bidel, P., Genel, N., Doit, C., Decre, D., Arlet, G. and Bingen, E. 2012. Phenotypic screening of carbapenemase and associated beta lactamases in carbapenem resistant Enterobacteriaceae. J. Clin. Microbiol., 50(4): 1295-1302.

CDC guidelines for control of Carbapenem resistant or Carbapenem producing Enterobacteriaceae in Acute care facilities. 2014.

Datta, P., Gupta, V., Garg, S. and Chander, J. 2012. Phenotypic method for differentiation of carbapenemases in Enterobacteriaceae: Study from north India. IJPM, 55(3): 357-360.

Gupta, E., Mohanty, S., Sood, S., Dhawan, B., Das, B.K. and Kapil, A. 2006. Emergence resistance to carbapenems in a tertiary care hospital in north India. Indian J. Med. Res., 124: 95-98.

Harbaik, J., Chudackova, E. and Papagiantis, C.C. 2014. Detection of carbapenemases in Enterobacteriaceae: a challenge for diagnostic microbiological laboratories. Clin. Microbiol. Infect., 20: 839-853.

Mate, H., Devi, S., Devi, M., Damrolien, S., Devi, N.L. and Devi, P.P. 2014. Prevalence of Carbapenem resistance in a Tertiary care hospital in North East India. J. Dent. Med. Sci., 13(12): 56-60.

Nagaraj, S., Chandran, S.P., Shamanna, P. and Macaden, R. 2012. Carbapenem resistance among Escherichia coli and Klebsiella pneumoniae in a tertiary care hospital in south India. Indian J. Med. Microbiol., 30: 93-95.

Nair, P.K. and Vaz, M.S. 2013. Prevalence of Carbapenem resistant 
Enterobacteriaceae from tertiary care hospital. J. Microbiol. Infect. Dis., 3(4): 201-210.

Nordaman, P., Naas, T. and Poirel, L. 2011. Global spread of carbapenemase producing Enterobacteriaceae. Emerg. Infect. Dis., 17(10): 1791-1798.

Okoche, D., Aslimwe, B.B., Katabazi, F.A., Kato, L. and Najjuka, C.F. 2015. Prevalence and characterization of carbapenem resistant Enterobacteriaceae isolated from Mulago National Referral Hoapital, Uganda. Plos One.

Praveen, M.R., Harish, B.N. and Parijaa, S.C. 2010. Emerging carbapenem resistance among nosocomial isolates of Klebsiella pneumoniae in south India. Int. J. Pharma and Bio Sci., 1(2).

Roy, S., Viswanathan, R., Singh, A.K., Das, P. and Basu, S. 2011. Sepsis in neonates due to imipenem resistant Klebsiella pneumoniae producing NDM-1 in India. J. Antimicrob. Chemother., Surinder kumar. Textbook of Microbiology. First edition. 2012. Jaypee brothers medical publishers.

Temple, D.K., Agarwal, J., Chaudhary, K., Lalwani, P., Tudu, M.S. and Hansdah, U. 2015. Carbapenem Resistance patterns in general intensive care unit of a Tertiary care hospital in India. $M A M C, 1(2)$ : 85-91.

Wang, X., Chen, G., Wu, X., Wang, L., Cai, J., Chan, E.W., et al. 2015. Increased prevalence of carbapenem resistant Enterobacteriaceae in hospital setting due to cross -species transmission of the bla NDM-1 $_{1}$ element and clonal spread of progenitor resistant strains. Frontier in Microbiol., vol 6.

Weinstien, R.A., Gupta, N., Limbago, B.M., Patel, J.B. and Kallen, A.J. 2011. Carbapenem resistant Enterobacteriaceae: Epidemiol. Prevention, Clin Inf. Dis., 53(1): 60-67.

Wattal, C., Goel, N., Oberoi, J.K., Raveendran, R., Datta, S. and Prasad, K.J. 2010. Surveillance of multidrug resistant organisms in a tertiary care hospital in Delhi, India. $J$. Assoc. Physicians India. 58:32-6.

\section{How to cite this article:}

Parimala, T.V. 2017. Screening of Carbapenem Resistant Enterobacteriaceae among Nosocomial Isolates: A study from South India. Int.J.Curr.Microbiol.App.Sci. 6(4): 460-465. doi: https://doi.org/10.20546/ijcmas.2017.604.053 